\title{
Abdominal Compartment Syndrome during Off-pump Coronary Artery Bypass: a Case Report
}

\author{
Yoon-Sook Lee ${ }^{1 *}$, Yong-Sang Yoon ${ }^{1}$, Soon-Young Hong ${ }^{1}$, Ae-Ran Park ${ }^{1}$, Yoon-Young Lee ${ }^{2}$ \\ ${ }^{1}$ Department of Anesthesiology and Pain Medicine, Korea University Ansan Hospital, Ansan, Korea \\ ${ }^{2}$ Department of Nursing, Sunchon National University, Suncheon, Korea
}

Received: September 22, 2016; Accepted: September 28, 2016, Published: September 29, 2016

*Corresponding author: Yoon-Sook Lee, Department of Anesthesiology and Pain Medicine, Korea University Ansan Hospita123, Jeokgeum-ro, Danwon-gu, Ansan-si, Gyunggi-do, 15355, South Korea, Tel: +82-31-412-5316; Fax: +82-31-412-5294; E-mail: yslee4719@gmail.com

\begin{abstract}
We report a case of intraoperative hemodynamic instability due to abdominal compartment syndrome in a 65-year-old woman who underwent elective off-pump coronary artery bypass graft. She had manifestation of destabilized hemodynamic and respiration, and detected an extremely tense and distended abdomen when the sternum was closed. After excluding hemorrhage and right heart failure, the patient was considered to have ACS. Then a nasogastric tube was inserted to decompress the stomach and $100 \mathrm{ml}$ of $20 \%$ mannitol was administered intravenously. With the above treatment, the patient was gradually relieved, and discharged 12 days later.
\end{abstract}

Keywords: Abdominal compartment syndrome; Off-pump coronary artery bypass graft surgery

\section{Introduction}

Multi vessel Off-Pump Coronary Artery Bypass Graft (OPCABG) is a practical and safe alternative to on-pump CABG[1]. Anesthetic management during OP-CABG is challenging because of hemodynamic instability that develops during the procedure. Abdominal Compartment Syndrome (ACS) is a path physiological consequence of increased Intra-Abdominal Pressure (IAP) that can be triggered by inflammation, hemorrhage, chemical peritonitis, or any other event that elevates IAP. This results in respiratory, renal, cardiac, or other organ dysfunction. We present a rare case of unexpected hemodynamic deterioration induced by ACS during OP-CABG.

\section{Case Report}

A 65-year-old woman (height $150 \mathrm{~cm}$, weight $55 \mathrm{~kg}$ ) was admitted for OP-CABG. Coronary angiography showed 50$80 \%$ stenosis of the left anterior descending artery, moderate atherosclerotic change in the left circumflex artery, and diffuse, severe atherosclerotic changes in the right coronary artery. Preoperative laboratory studies were unremarkable.

Anesthesia was induced, and intraoperative hemodynamic was relatively stable until completion of the anastomoses. When the surgeons commenced bleeding control, blood pressure dropped from $110 / 70$ to $60 / 30 \mathrm{mmHg}$ and heart rate increased from 80 to $130 / \mathrm{min}$. At a tidal volume of $400 \mathrm{ml}$ and a respiratory rate of $12 / \mathrm{min}$, peak airway pressure concomitantly increased from 18 to $30 \mathrm{~cm} \mathrm{H}_{2} \mathrm{O}$, end tidal $\mathrm{CO}_{2}$ increased from 29 to 33 $\mathrm{mmHg}$, and $\mathrm{SpO}_{2}$ in $50 \%$ oxygen/air mixture decreased to $96 \%$. Lung sounds were equal and clear. Then, Mean Pulmonary Arterial Pressure (mPAP) increased from 18 to $28 \mathrm{mmHg}$, and central venous pressure (CVP) increased from 5 to $17 \mathrm{mmHg}$. Fluid was infused rapidly and inotropic support was provided, including dopamine, dobutamine, and phenylephrine. Phenylephrine (50 $\mu \mathrm{g})$ was administered intravenously. Phenylephrine (100 $\mu \mathrm{g})$ was then given repeatedly, which increased blood pressure only slightly to $90 / 50 \mathrm{mmHg}$. Dopamine and dobutamine were titrated to maintain systolic blood pressure $>90 \mathrm{mmHg}$. Blood pressure was maintained at 90-100/50-60 mmHg and heart rate was maintained at $110-130 / \mathrm{min}$. Urinary output was maintained during the operation. An arterial blood gas analysis on $100 \%$ oxygen showed $\mathrm{pH}$ of $7.25, \mathrm{PaCO}_{2}$ of $45.1 \mathrm{mmHg}$, and $\mathrm{PaO}_{2}$ of 124 $\mathrm{mmHg}$. Tidal volume and respiratory rate were reset to improve the progressively worsening oxygenation and to increase peak inspiratory pressure. CVP and mPAP did not decrease during fluid resuscitation and inotropic support. Bleeding and right heart failure was suspected. The surgeons were informed that ventilation was becoming more difficult, but no cause for the problem was provided. Hemoglobin was $10.2 \mathrm{~g} / \mathrm{dl}$ and platelet count was 55,000 cells/ $\mu$ l. The patient had received about 2000 $\mathrm{ml}$ of intravenous crystalloid solution, five units of packed red blood cells, five units of fresh frozen plasma, and eight units of platelet concentrate.

An extremely tense and distended abdomen was detected when the sternum was closed. A nasogastric tube was inserted to decompress the stomach and $100 \mathrm{ml}$ of $20 \%$ mannitol was administered intravenously. Respiratory and the hemodynamic variables improved gradually $30 \mathrm{~min}$ later. Peak inspiratory pressure decreased to $25 \mathrm{~cm} \mathrm{H}_{2} \mathrm{O}$, blood pressure increased to $110 / 60 \mathrm{mmHg}$, and heart rate decreased $100 / \mathrm{min}$. Ultrasonography revealed fluid in the abdominal cavity; the kidneys appeared normal, and the bowel loops were edematous 
(Figure 1). Because surgical bleeding was suspected, diagnostic paracentesis was performed under ultrasonographic guidance immediately after skin closure. Fluid was detected in the abdomen by sonography and approximately $50 \mathrm{ml}$ of clear yellow fluid was aspirated. At the end of surgery, blood pressure was restored to 120-140/70-80 mmHg, and heart rate had decreased to 70-80/ min. An arterial blood gas analysis on $100 \%$ oxygen showed $\mathrm{pH}$ of 7.327, $\mathrm{PaCO}_{2}$ of $33.7 \mathrm{mmHg}$, and $\mathrm{PaO}_{2}$ of $139.2 \mathrm{mmHg}$.

The patient underwent an abdominal contrast-enhanced Computed Tomography (CT) scan. $\mathrm{SpO}_{2}$ was $100 \%\left(\mathrm{FiO}_{2}=1.0\right)$ upon arrival in the intensive care unit. Peak inspiratory pressure had decreased to $20-25 \mathrm{~cm} \mathrm{H}_{2} \mathrm{O}$. An arterial blood gas analysis on $60 \%$ oxygen showed $\mathrm{pH}$ of $7.497, \mathrm{PaCO}_{2}$ of $27.7 \mathrm{mmHg}$, and $\mathrm{PaO}_{2}$ of $275 \mathrm{mmHg}$. A complete blood count and serum electrolytes were within normal ranges. The abdominal CT showed a diffuse edematous bowel wall with adjacent fluid but no detectable left ureteral contrast media due to a suspicious thickened left pelvoureteral junction (Figure 2). Her postoperative course was uneventful, and the patient was discharged from the hospital 12 days after surgery without any postoperative complications.

\section{Discussion}

We encountered a case of hemodynamic deterioration

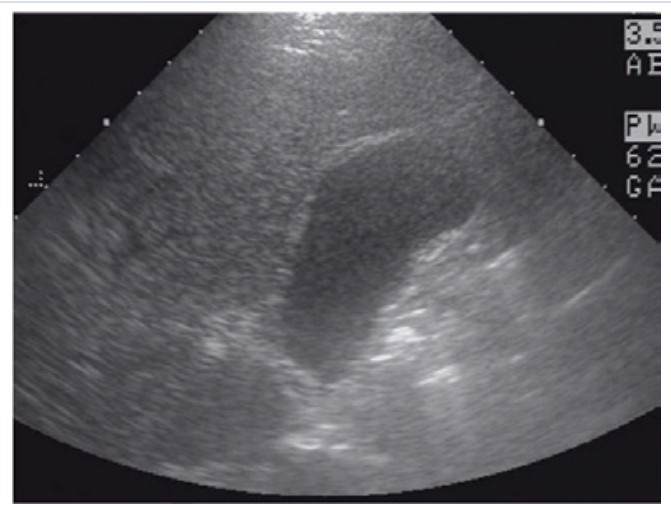

Figure 1: Portable abdominal sonography. Fluid collection in the pelvic cavity and right side of the paracolic gutter

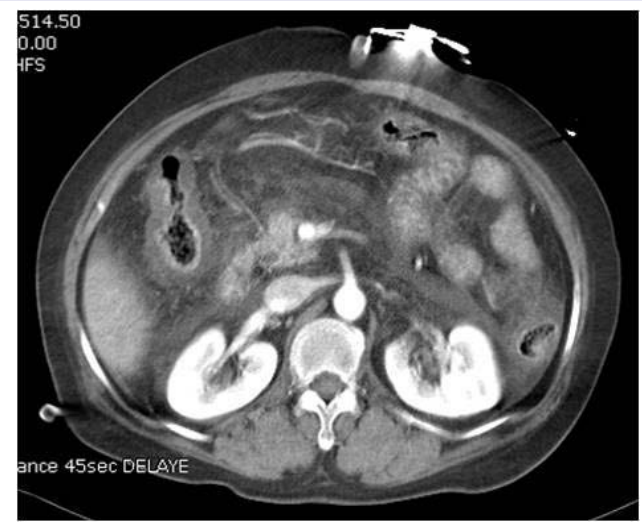

Figure 2: Contrast-enhanced abdominal computed tomography showing edematous and inflammatory bowel changes with adjacent collection of fluid with exacerbation, which required intensive hemodynamic management induced by ACS during OP-CABG.

ACS causes significant dysfunction of almost all organ systems. The increase of Intra-Abdominal Pressure (IAP) in a patient with ACS decreases lung compliance and increases peak airway pressure during mechanical ventilation, resulting in hypoxemia and hypercapnia. It also decreases venous return, which reduces cardiac output and abdominal organ perfusion, causing oliguria and splanchnic ischemia [2,3]. Normal IAP is $<0 \mathrm{mmHg}$, and IAP $>20 \mathrm{mmHg}$ is clinically significant. Recent studies have demonstrated that significant alterations in organ function occur even at a relatively low IAP of 10-15 $\mathrm{mmHg}[4,5]$. We could not measure IAP but we knew that the abdominal wall was distended, and the small bowel loops appeared edematous. Experiments with bowel mucosa show decreased oxygenation and worsening acidosis in mucosal cells exposed to IntraAbdominal Hypertension [6]. Therefore, an indirect diagnosis of ACS was made in our case.

Hemodynamic deterioration during OP-CABG can be secondary to myocardial ischemia, reduced preload, myocardial dysfunction, compression of cardiac chambers, mitral regurgitation, or a combination of these [7]. Because our patient showed an abrupt increase in pulmonary arterial pressure and CVP and there was no ischemic ST-T change on the electrocardiogram or mitral regurgitation during the perioperative period, the possible cause was myocardial dysfunction or compression of the cardiac chambers. Patients with ACS typically present with a tense distended abdomen, increased peak inspiratory airway pressures, severe hypercapnia, hypotension, and oliguria [8]. Decreasing tidal volume, increasing respiratory rate and applying positive-end expiratory pressure were applied to reset these parameters to improve the worsening oxygenation and increase peak inspiratory pressure. The crisis lasted for one and a half hours and then the surgeons closed the sternum. The patient's hemodynamics was then stably maintained until the end of surgery.

Acute ACS can be a difficult clinical diagnosis, as anesthesiologists must practice a high index of suspicion in the differential diagnosis of hemodynamic compromise and hemorrhagic shock. In this case, ACS could have been exacerbated by administering a massive volume of fluid that contributed to ascites and abdominal wall edema. The key is early detection and efforts to avoid aggressive fluid resuscitation. The associated rise in abdominal pressure increases the resistance to venous return, which reduces venous return leading to low cardiac output and shock [9]. Furthermore, as IAP increases, the diaphragm is pushed cephalad, which reduces thoracic or extrapulmonary compliance. The clinical manifestations of ACS are non-specific and include decreased urinary output, increased ventilatory pressures, and a tense abdomen. Specific treatment goals are to improve abdominal wall compliance, reduce the volume of abdominal fluid, and correct the positive fluid balance. Abdominal fluid collection is generally drained percutaneously under radiological guidance. 
Anesthesiologists have difficulty to interpret hemodynamics of patients with ACS. Because of increased abdominal pressure, CVP and pulmonary artery occlusion pressure are falsely elevated and do not show true cardiac preload. The main limitations of OP-CABG are incomplete revascularization and intraoperative hemodynamic instability. Therefore, we suspected intraoperative surgical bleeding and right heart failure but did not consider ACS. We emphasize that all anesthesiologists and surgeons must be aware of ACS during OP-CABG, as this could be a potential complication leading to significant hemodynamic deterioration if not recognized.

\section{References}

1. Abu-Omar Y, Taggart DP. The present status of off-pump coronary artery bypass grafting. Eur J Cardiothorac Surg. 2009;36(2):312-321. doi:10.1016/j.ejcts.2009.03.025.

2. An G, West MA. Abdominal compartment syndrome: a concise clinical review. Crit Care Med. 2008;36(4):1304-1310. doi:10.1097/ CCM.0b013e31816929f4.
3. Deenichin GP. Abdominal compartment syndrome. Surg Today. 2008;38(1):5-19.

4. Ridings PC, Bloomfield GL, Blocher CR, Sugerman HJ. Cardiopulmonary effects of raised intra-abdominal pressure before and after intravascular volume expansion. J Trauma. 1995;39(6):1071-1075.

5. Bongard F, Pianim N, Dubecz S, Klein SR. Adverse consequences of increased intra-abdominal pressure on bowel tissue oxygen.J Trauma. 1995;39(3):519-524.

6. Bailey J, Shapiro MJ. Abdominal compartment syndrome. Crit Care. 2000;4:23. DOI:10.1186/cc646.

7. Couture P, Denault A, Limoges P, Sheridan P, Babin D, Cartier R. Mechanisms of hemodynamic changes during off-pump coronary artery bypass surgery. Can J Anaesth. 2002;49(8):835-849.

8. Hunter JD, Damani Z. Intra-abdominal hypertension and the abdominal compartment syndrome. Anaesthesia. 2004;59(9):899-907.

9. Sugrue M. Abdominal compartment syndrome. Curr Opin Crit Care. $2005 ; 11(4): 333-338$ 